Palenzuela, P.; Olivi, A. (coords.) (2011). Etnicidad y desarrollo en los Andes. Sevilla, Universidad de Sevilla, Secretariado de Publicaciones.

\title{
Etnicidad y desarrollo en los Andes
}

Jaume Vallverdú Vallverdú

Departamento de Antropología, Filosofía y Trabajo Social, URV

jaime.vallverdu@urv.cat

Esta obra surge de las aportaciones a un Seminario Internacional con el mismo título celebrado en la Universidad de Sevilla en noviembre de 2009. Reúne un total de nueve artículos de enfoque antropológico sobre la temática, que a continuación se sintetizan según la misma secuencia del libro.

El texto de Joan Josep Pujadas sirve de contextualización teórica preliminar a la categoría "etnia" o "grupo étnico", y más en particular a la validez de su uso para la etnografía y la interpretación de la diversidad cultural. Resume las principales aportaciones teóricas contemporáneas al debate sobre la etnicidad —la culturalista y la constructivista- y propone alternativas conceptuales para su tratamiento empírico en el contexto de las sociedades multiculturales. Sigue a esto la presentación del estudio etnográfico del autor sobre la evolución histórica del significado social de la frontera en la Cerdaña (Pirineo catalán). Se explica de quée modo la frontera se convierte en una clara marca de diferenciación: de ser tradicionalmente compartida, pasará a dividir dos modelos de sociedad en el marco de dos proyectos políticos nacionales —el francés y el españolsin nada en común. Posteriormente, Pujadas sugiere revisar la categoría "grupo étnico" como unidad analítica a la luz del papel hegemónico habitualmente ejercido por el Estado sobre grupos subordinados o minoritarios. Propone que se activen cambios legislativos o constitucionales que acaben con las históricas prácticas estatales etnocidas, etnofágicas, de asimilación forzada o exclusión de las poblaciones étnica o racialmente diferentes. El nuevo paradigma de la etnicidad requeriría primeramente una crítica al etnocentrismo que, según $\mathrm{Pu}$ jadas, sigue dominando el pensamiento racional de las élites de todo el mundo, incluida la intelectual, e implicaría a la vez — siguiendo a Maybury-Lewis - la necesidad de construir "una nueva teoría comprensiva de los estados multiétnicos que revise [...] el concepto de integración, que suplanta las conciencias 
colectivas, que minimiza el componente civilizatorio del legado o de las comunidades étnicas y que infantiliza, exotiza (o, simplemente, excluye de raíz) las voces de sus actores" (pág. 46).

Pablo Palenzuela analiza a continuación los discursos y las prácticas de los actores implicados en el proyecto de desarrollo rural PRODECO, activado en Cotopaxi (República del Ecuador) y promovido por la Unión Europea (UE) entre 2002 y 2007. Subraya la importancia, por una parte, de considerar la naturaleza política de todo proceso de desarrollo, dando protagonismo central al sujeto del mismo y a su toma de decisiones, y por otra, de "identificar las estructuras de poder que detentan la capacidad de decidir y monopolizar, o comparten, el control del proceso" (pág. 55). Así, entiende que todo desarrollo endógeno debe complementarse de forma ideal con los recursos exógenos considerados apropiados, siempre y cuando estos sean sometidos al "control cultural” que requiere el etnodesarrollo, según lo define Bonfill Batalla. Palenzuela asegura, además, que el "desarrollismo", teñido de etnocentrismo, eurocentrismo y economicismo tecnocrático, sigue siendo el paradigma en el que se inspiran la mayoría de las acciones de cooperación al desarrollo, o de las prácticas surgidas del llamado "desarrollo institucionalizado". Frente a este modelo hegemónico, funcional al criterio de acumulación neoliberal-capitalista, al poder de los países centrales sobre los periféricos y a la reificación de los esquemas de la modernización occidental, se reivindica el papel de los análisis interdisciplinares - en especial entre la economía política, la historia y la antropología social一, así como la pertinencia analítica de los enfoques del conflicto e historicista. A tenor de los resultados del proyecto PRODECO, se reconoce la imposibilidad de verificar etnográficamente el objetivo de promover un desarrollo con identidad y sostenible en el medio indígena, donde finalmente son las agencias institucionales y las ONG las que acaban decidiendo según sus criterios y conveniencias el modelo y los procedimientos de la cooperación al desarrollo en la provincia. De este modo, sin el control cultural de los procesos de implementación de los proyectos y de los recursos que se aplican a la cooperación al desarrollo, el movimiento indígena se vería despojado de incidencia política real y efectiva en Cotopaxi.

El siguiente artículo es el de Luis Alberto Tuaza, enfocado hacia los procesos políticos y de desarrollo económico en los que se han visto implicados los indígenas de Columbe, en la provincia de Chimborazo. El autor empieza 
describiendo la acción de los primeros organismos de cooperación en la zona a partir de la segunda mitad del pasado siglo, para contextualizar después, desde los años ochenta en adelante, "el apogeo de los ideales de desarrollo" materializados en diferentes proyectos de índole gubernamental, civil o religiosa. Según Tuaza, el discurso global de las ONG no se corresponde con la realidad de las comunidades y los indígenas siguen siendo considerados incapaces de promover su propio desarrollo. De todo ello se derivan, además, procesos de fragmentación y "cansancio organizativo" de las comunidades, el debilitamiento de las agrupaciones indígenas de base y, en suma, la ruptura de las formas tradicionales de vida a partir de las imposiciones y los criterios de orden externo. Finalmente, se propone la necesidad de suscitar el debate tanto sobre los planes gubernamentales y no gubernamentales de desarrollo como sobre los discursos y las prácticas que revelan, buscando su conexión con la realidad, tomando a los sujetos destinatarios como auténticos protagonistas de su presente y futuro y desmitificando, si es necesario, la noción misma de desarrollo para proponer programas y proyectos coherentes, eficientes y no desvirtuados por el clientelismo y el paternalismo.

Víctor Bretón estudia en Toacazo (Cotopaxi) las bases constitutivas de la Confederación de Nacionalidades Indígenas del Ecuador (CONAIE). El punto de partida de la reflexión en clave histórica sobre este proceso son las reformas agrarias de los años sesenta y setenta y, más en particular, el fin del régimen de la hacienda, elemento vertebrador de un sistema de dominación bien estructurado y conformado en las tierras altas ecuatorianas desde el siglo XVII como un engranaje de explotación del campesinado indígena, de legitimación del orden existente y dispositivo principal de los mecanismos de control y gobierno de las poblaciones. En relación con las dinámicas de la hacienda se señalan, entre otros aspectos, la existencia de conflictos endógenos, el paternalismo del terrateniente y el "gamonalismo", referido a cómo la hacienda se articulaba verticalmente en los niveles parroquiales y cantonales de dominación étnica, determinando un orden social asentado en la combinación de la coerción y el consenso que se prolongó hasta los años setenta del pasado siglo. En este contexto, el autor se sirve del concepto clásico de hegemonía de Antonio Gramsci para explicar el surgimiento de un nuevo "sujeto político" indígena organizado, resistente y reivindicativo. Se destacará aquí el papel decisivo de los intelectuales orgánicos vinculados al pensamiento social y religioso de izquierdas para 
encauzar el final del régimen de la hacienda en la zona andina. Esto supondrá, según el autor, el establecimiento de un nuevo marco de relaciones con el Estado, así como la formación de una nueva élite intelectual indígena organizada y capaz de forjar alianzas de oposición a los terratenientes a diferentes niveles.

Otro texto que reflexiona sobre el movimiento indígena de Ecuador es el de Carmen Martínez, quien reconoce en primer lugar el impacto legislativo internacional que las teorías del etnodesarrollo han tenido en relación con los derechos de los pueblos indígenas. La extensión del concepto de etnodesarrollo a distintos países de Iberoamérica habría promovido, además, su versión más reciente: el llamado "buen vivir", noción resultante del activismo político indígena en las últimas décadas y que exalta la armonía del ser humano con la naturaleza, el desarrollo basado en la calidad de vida, un sistema económico solidario, la diversidad cultural, etc. Martínez se pregunta, no obstante, si las políticas ecuatorianas de derechos indígenas suponen realmente un proceso de desarrollo que busca el "buen vivir" en formas alternativas. Concluirá que, debido al carácter autoritario y centralista del gobierno vigente, el precepto básico de este concepto no se está cumpliendo, a saber: el control de los pueblos indígenas de sus recursos naturales y la capacidad de decidir sobre su propio desarrollo. En último término, el ideal del "buen vivir" es considerado más bien un elemento discursivo destinado a legitimar un proyecto político que puede permitir la reproducción cultural — es decir, ser un nuevo tipo de "indigenismo no asimilacionista" - sin que el propio grupo étnico tenga el control cultural de este proceso.

Posteriormente, Eduardo Kingman, al analizar la realidad indígena en medio urbano, describe las transformaciones del barrio popular de San Roque, en el centro histórico de Quito. El autor explica de qué modo después de una larga historia de olvido e indiferencia respecto a sus condiciones ambientales, económicas, sociales y de seguridad, San Roque se vuelve un "lugar deseado", objeto de singular interés y preocupación civil. Integrado básicamente por población indígena y mestiza, el barrio en cuestión se presenta como un caso, entre otros, de este significativo cambio perceptivo. De ahí que Kingman se interese no solamente por saber lo que se intenta hacer con esos sitios sino por "el sentido mismo de esa nueva forma de pre-ocuparse por ellos" (pág. 202). Para el autor se trata de una pre-ocupación perversa, puesto que se relaciona de alguna forma con ciertas políticas de intervención, o más concretamente, de 
intervención patrimonial. Analizando la relación existente entre las acciones de renovación urbanística y las prácticas de patrimonialización, se observarán ante todo los intereses específicos de los planificadores urbanos y de los especuladores inmobiliarios, agentes en su mayoría externos a la zona y desconocedores de sus sensibilidades y problemáticas. Al mismo tiempo, nos dice Kingman, la recuperación y revalorización del patrimonio se relaciona directamente con políticas de seguridad, de modo que las demandas de intervención patrimonial dan lugar a acciones higienistas y de control policial. El autor señala al respecto que es necesario el estudio crítico del modelo y de la práctica de seguridad que suelen acompañar a los procesos de transformación urbana descritos, en la medida en que activan graves procesos de exclusión y estigmatización social. Políticas cualitativamente diferentes deben ser aquellas que tengan en consideración las demandas y necesidades de la gente, rehabilitando los espacios sin promover su expulsión.

Alessandra Olivi examina a continuación la construcción de los proyectos vital y político de los mapuches chilenos a la luz del persistente conflicto sobre sus derechos territoriales, actualmente "latentes o amenazados". Con las herramientas de la antropología fenomenológica y de la economía política, Olivi se acerca a la vivencia y construcción del lugar por referencia tanto a los procesos de significación y vinculados a la identidad como a las resistencias frente a las dinámicas capitalistas y sus fuerzas globales. Para el caso de los mapuches chilenos, afirma que dicha construcción y su validez política "descansa en la capacidad de las personas de seguir generando dinámicas de reciprocidad identitaria con el territorio que habitan, como sustento material y simbólico de la propia especificidad cultural" (pág. 228). La última parte del texto recupera la contradicción fundamental que se establece entre el reconocimiento legal de los derechos de los mapuches sobre sus tierras y la presión económica que representa la penetración en ellas de las empresas privadas capitalistas, con sus nefastas consecuencias ecológicas sobre los territorios indígenas. En tales circunstancias, cada vez será mayor la dificultad que tienen las comunidades indígenas para responder a los cambios impuestos por las fuerzas exteriores y los poderes globales — capital, Estado, tecnología - guiados por la lógica del mercado. La histórica capacidad de adaptación mapuche al impacto de los procesos de dominación se ve ahora gravemente desafiada, como debilitada se ve también la fuerza reivindicativa del movimiento social e indígena en relación a sus dere- 
chos territoriales y culturales. Alessandra Olivi concluirá que no puede haber soluciones para el problema indígena desde la óptica de un Estado que rechaza la realización de proyectos de vida alternativos a la visión hegemónica. Sin esa voluntad política no se puede satisfacer la demanda básica de los mapuches: "la restauración de posibilidades efectivas de relacionarse con un ecosistema determinado, relación que está en la base de su reproducción cultural y la cual se funda en la noción de territorio indígena” (pág. 249).

El siguiente texto es el de Andreu Viola, centrado de nuevo en la relevancia para el mundo andino actual del concepto del "buen vivir" (Sumaq Kawsay) en su relación con los procesos de desarrollo e identitarios del mundo indígena. No en vano es una noción que incluye la armonía con el entorno social (la comunidad), con el entorno ecológico (la naturaleza) y con el entorno sobrenatural, y que está al mismo tiempo estrechamente vinculada a la concepción de la persona, su capacidad de trabajo y el comunitarismo. Viola explica cómo las concepciones del "buen vivir" se han incorporado a las Constituciones de Ecuador y de Bolivia, aunque no sin ciertas imprecisiones y ambigüedades importantes que afectarían a su aplicación práctica. Junto a este inconveniente se señala, además, un riesgo asociado a la idealización o instrumentalización del concepto: la pérdida de su significado, y con ello, de su mismo potencial transformador. El autor apunta, en este sentido, una tendencia a la mistificación del "buen vivir" por referencia a toda una serie de actores y agentes externos al campesinado andino - ONG, intelectuales en su mayoría no indígenas, asesores y consultores internacionales - cuyo apoyo a la diversidad cultural y a las demandas de los pueblos indígenas puede llevar, contradictoriamente, "a la adopción de planteamientos idealizados, irreales y esencialistas sobre sus necesidades, sus aspiraciones $y$, en definitiva, sobre su modus vivendi [...], minimizando sus tensiones internas y las serias amenazas que se ciernen sobre [el mundo rural andino] en la actualidad" (págs. 271 y 129). Viola sostendrá que dicho mundo debería dejar de ser percibido desde el imaginario occidental "como un 'vacío' a rellenar y completar desde el exterior, por parte de legiones de técnicos y cooperantes actuando como 'mercaderes' de Progreso” (pág. 294), expresando, sobre esa base, una toma clara de posición que devuelve el protagonismo a los indígenas y al control cultural de sus procesos de vida, organización y trabajo. 
El último artículo del volumen se ubica empíricamente en la cuenca baja del río Curaray (Amazonía ecuatoriana). En él, Javier Martínez analiza la interacción entre las políticas ambientalistas y los pueblos indígenas a partir del caso de un Proyecto de Conservación de la Biodiversidad financiado por el Banco Mundial y ejecutado por la ONG indígena Instituto Quichua de Biotecnología Sacha Supray. Según Martínez, la apropiación e interiorización del discurso del ecologismo y de su plasmación en proyectos de "desarrollo sostenible" por parte de las organizaciones indígenas habría desembocado en la construcción de "identidades ecológicas" o "eco-identidades", las cuales, afirma, están en la base de su estrategia política para asegurar el acceso a los recursos económicos y hacer valer sus derechos territoriales. Para aumentar, en definitiva, su poder político, "volviéndose un poderoso medio para formular reivindicaciones territoriales" (pág. 319). Esta estrategia se ve además reforzada, en primer lugar, porque responde a las políticas y a los intereses de las grandes agencias de desarrollo que operan en la zona y, secundariamente, porque favorece la consolidación de redes con un número creciente de organizaciones ambientalistas que comparten con las organizaciones indígenas locales la preocupación por el estado del planeta. Por otro lado, la necesaria contextualización política y económica de la zona objeto de estudio remite al triángulo desarrollo-etnicidadmedio ambiente. $Y$, en particular, a estudiar la forma en que se ha desarrollado un discurso en el que los temas ambientales y étnicos conforman dos pilares fundamentales de las demandas indígenas. En este ámbito, la relación entre las organizaciones indígenas y las ONG ambientalistas y ecologistas se observa a la luz del proceso de construcción del llamado "nativo ecológico", el cual, siendo al fin y al cabo "un instrumento de los organismos de conservación y de las organizaciones ecologistas", es a la vez "un medio para formular las reivindicaciones identitarias, culturales y políticas de las organizaciones indígenas" (págs. 317-318). Con todo, Martínez no ve al indígena como objetivamente resistente y emancipador, sino más bien como un sujeto eventualmente implicado en procesos de cooptación y, en cualquier caso, muy inmerso en el sistema de las políticas hegemónicas. Todo ello aunque al mismo tiempo se valga, de modo estratégico, de la identidad ecológica o eco-identidad para formular de manera legítima reivindicaciones políticas y económicas, muchas de ellas de carácter territorial. 
Valorando por separado y de forma conjunta todas las aportaciones hasta aquí presentadas, podemos decir que el libro reseñado constituye una excelente contribución al tema objeto de estudio. Ofrece una útil panorámica general y contextual y constituye al mismo tiempo un buen caleidoscopio etnográfico en torno a los procesos de cambio social y cultural vinculados a la realidad indígena en los Andes. 